\title{
Radio afterglow of the jetted tidal disruption event Swift J1644+57
}

\author{
B.D. Metzger ${ }^{1, a}$, D. Giannios ${ }^{1}$, and P. Mimica² \\ ${ }^{1}$ Department of Astrophysical Sciences, Peyton Hall, Princeton University, Princeton, NJ 08544, USA \\ 2 Departmento de Astronomia y Astrofisica, University de Valencia, 46100 Burjassot, Spain
}

\begin{abstract}
The recent transient event Swift J1644+57 has been interpreted as resulting from a relativistic outflow, powered by the accretion of a tidally disrupted star onto a supermassive black hole. This discovery of a new class of relativistic transients opens new windows into the study of tidal disruption events (TDEs) and offers a unique probe of the physics of relativistic jet formation and the conditions in the centers of distant quiescent galaxies. Unlike the rapidly-varying $\gamma / \mathrm{X}$-ray emission from Swift J1644+57, the radio emission varies more slowly and is well modeled as synchrotron radiation from the shock interaction between the jet and the gaseous circumnuclear medium (CNM). Early after the onset of the jet, a reverse shock propagates through and decelerates the ejecta released during the first few days of activity, while at much later times the outflow approaches the self-similar evolution of Blandford and McKee. The point at which the reverse shock entirely crosses the earliest ejecta is clearly observed as an achromatic break in the radio light curve at $t \approx 10$ days. The flux and break frequencies of the afterglow constrain the properties of the jet and the $\mathrm{CNM}$, including providing robust evidence for a narrowly collimated jet. I briefly discuss the implications of Swift J1644+57 for the fraction of TDEs accompanied by relativistic jets; the physics of jet formation more broadly; and the prospects for detecting off-axis TDE radio emission, either via follow-up observations of TDE candidates discovered at other wavelengths or blindly with upcoming wide-field radio surveys. The radio rebrightening observed months after the onset of the jet remains a major unsolved mystery, the resolution of which may require considering a jet with more complex (temporal or angular) structure.
\end{abstract}

\section{INTRODUCTION}

A rare glimpse into the properties of normally quiescent supermassive black holes (SMBHs) is afforded when a star passes sufficiently close that it is tidally disrupted. Numerical simulations of 'tidal disruption events' (TDEs) show that a significant fraction of the shredded star remains gravitationally bound to the black hole (e.g. Ayal et al. 2000; Guillochon et al. 2009). Accretion of this stellar debris has long been predicted to power a thermal 'flare' at optical, UV, and X-ray wavelengths that lasts for months to years after the merger (e.g. Lodato et al. 2009; Strubbe \& Quataert 2009).

Giannios \& Metzger (2011; GM11) explore the consequences if a modest fraction of the accretion power from a TDE is used to accelerate a collimated jet to ultra-relativistic speeds. Such a short-lived ejection cannot propagate far from the SMBH before beginning to interact and decelerate via its interaction with the surrounding circumnuclear medium (CNM). GM11 showed that the jetCNM interaction is mediated by shocks, which powers synchrotron emission peaking on a timescale $\sim$ monthsyears after the merger. An alternative model for radio emission from TDE jets was developed by Van Velzen et al. (2011), who instead focused on emission internal to the jet itself by making a phenomenological connection with the radio/X-ray correlations of stellar mass compact binaries.

The gamma-ray transient Swift J164449.3+573451 (hereafter Sw J1644+57) was detected by the Swift/BAT on March 25, 2011. Subsequent imaging at radio, optical, and $\mathrm{X}$-ray wavelengths localized the event to within $\lesssim 100 \mathrm{pc}$ of the center of a compact galaxy at redshift $z \simeq 0.35$

a e-mail: bmetzger@phys.columbia.edu (e.g. Berger et al. 2011). The coincidence of Sw J1644+57 with the galactic nucleus, combined with the lack of previously known GRBs with similar luminosity or duration (Levan et al. 2011), suggest that it most likely originated from a rapid onset of accretion onto a $\mathrm{SMBH}$, as probably can only be explained by a TDE (Bloom et al. 2011; see Levan, Zauderer, this conference). The SED of Sw J1644+57 showed two distinct components, suggesting different sources for the X-ray and radio emission. The high energy emission is rapidly variable, placing its origin at small radii close to the SMBH, likely from a location 'internal' to the jet itself. Constraints on the brightness temperature instead place the radio emission at much larger radii, suggesting that it results from the shock interaction of the jet with the CNM, as originally predicted by GM11 just months prior to the discovery of $\mathrm{Sw}$ J1644+57.

\section{JET-CNM INTERACTION}

\subsection{Evidence for the reverse shock crossing}

Metzger, Giannios, \& Mimica (2012; MGM12) model the radio emission from $\mathrm{Sw} \mathrm{J} 1644+57$ in order to constrain the properties of the relativistic jet and the CNM surrounding the SMBH, using the observed X-ray luminosity as a proxy for the jet power. The X-ray light curve shows an initial period of several bright flares lasting for a duration $t_{\mathrm{j}} \sim$ several days, before declining as a power-law in a manner roughly consistent with the expectations of fallback accretion (Rees 1988) if the jet emission were to directly track the accretion rate.

Interaction between the matter in the relativistic jet and the CNM occurs in two stages: (1) Initially, at times 


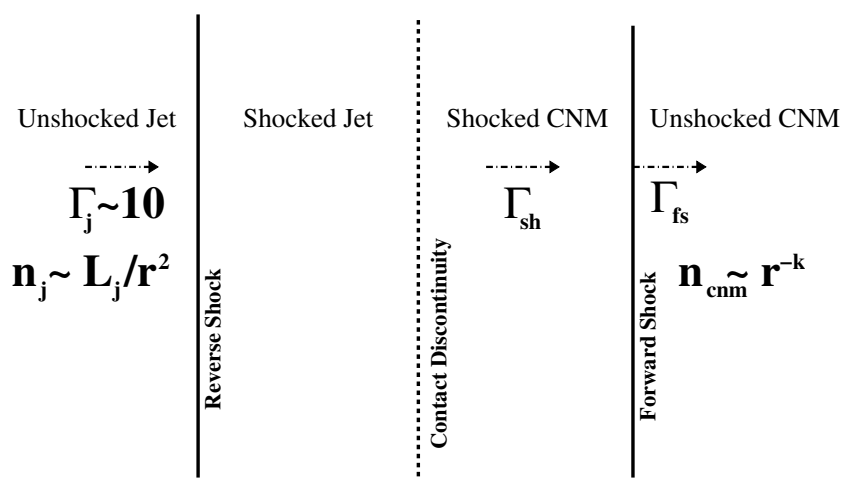

Figure 1. Schematic illustration of the forward-reverse shock structure produced by the interaction of a relativistic jet and the surrounding circumnuclear medium during the early phase $\left(t \lesssim t_{\mathrm{j}}\right)$ when the reverse shock crosses back through the shell of ejecta released during the initial period of jet activity (from MGM12).

$t \lesssim t_{\mathrm{j}}$, the jet drives a forward shock (FS) into the CNM, while simultaneously a reverse shock (RS) propagates back through the ejecta (Fig. 1). (2) At later times, once the RS has entirely crossed through the initial ejecta $\left(t \gg t_{\mathrm{j}}\right)$, the blastwave approaches a self-similar expansion (e.g. of Blandford \& McKee 1976; BM76).

The transition between stages (1) and (2) decreases the pressure of the shocked fluid, starting from behind the RS. The pressure drop is then communicated after a delay to the FS by a rarefaction that is launched from the back of the shocked shell. This signal propagates at the (relativistically hot) sound speed of the shocked fluid, reaching the FS at an observer time $t \sim 2 t_{\mathrm{j}}$, i.e. about twice the observed duration of peak jet activity. Figure 2 shows the evolution of the Lorentz factor $\Gamma_{\mathrm{sh}}$ of material at various locations within the shocked ejecta (measured in the lab frame), as calculated using a one-dimensional relativistic hydrodynamic simulation of a jet with a constant (isotropic) kinetic luminosity $L_{j, 0}=10^{48} \mathrm{ergs}^{-1}$, duration $t_{\mathrm{j}}=3 \times 10^{5} \mathrm{~s}$, and initial Lorentz factor $\Gamma_{\mathrm{j}}=10$ (jet spreading is relatively minor as long as the jet is ultrarelativistic; e.g. Zhang \& MacFadyen 2009). Note that a break occurs once the RS has crossed the ejecta, first immediately behind the $\mathrm{RS}\left(t \approx t_{\mathrm{j}}\right)$ and then later behind the FS $\left(t \sim 2 t_{\mathrm{j}}\right)$. As we now discuss, this last break in the Lorentz factor produces an achromatic break in the resulting radio light curve since emission from the FS dominates that from the RS in the case of Swift J1644+57 (although see §3.4).

Figure 3 shows the radio light curves of Sw J1644+57 from Zauderer et al. (2011) at several frequencies below the self-absorption frequency. The data are well fit by a power law $F_{v} \propto t^{\alpha}$ with $\alpha=2$ at times $t \lesssim t_{\text {break }}=10$ days, followed by a break to shallower rise $\alpha \approx 0.5$ at late times. As discussed above, this achromatic break is naturally explained as the rarefaction reaching the FS following the RS crossing the ejecta; the time of the break at $t_{\text {break }} \approx 2 t_{\mathrm{j}} \sim 10$ days is consistent with this picture (compare Figures 2 and 3). Alternative explanations for the observed break (such as the 'jet breaks' in normal GRB afterglows) are discussed by MGM12 and ruled out.

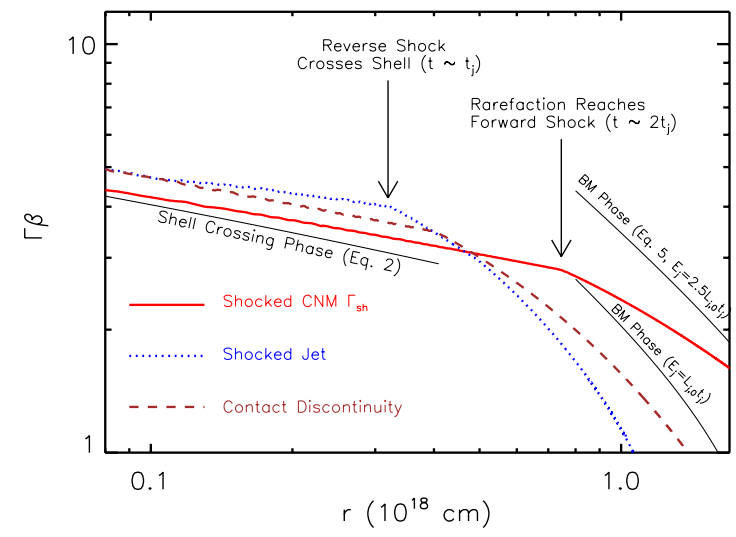

Figure 2. Lorentz factor of the shocked circumnuclear medium (CNM) behind the forward shock ( $\Gamma_{\mathrm{sh}}$; thick solid red line), of the contact discontinuity (dashed brown line) and of the shocked jet (dotted blue line), extracted directly from a one-dimensional hydrodynamic simulation (Mimica et al. 2009). The calculation is performed assuming a jet duration $t_{\mathrm{j}}=3 \times 10^{5} \mathrm{~s}$, initial jet luminosity $L_{j, 0}=10^{48} \mathrm{ergs} \mathrm{s}^{-1}$ and CNM radial density profile $n_{\mathrm{cnm}}=10\left(r / 10^{18} \mathrm{~cm}\right)^{-1} \mathrm{~cm}^{-3}(k=1)$. Shown for comparison with black solid lines are the analytic approximations for $\Gamma_{\mathrm{sh}}$ during the early phase when the reverse shock is crossing through the shell of ejecta released during the initial period of constant jet luminosity $\left(t \lesssim t_{\mathrm{j}}\right)$, and at late times during the Blandford-McKee self-similar evolution $\left(t \gg 2 t_{\mathrm{j}}\right.$ ) for different assumptions about the total energy of the blast wave $\mathrm{E}_{j, i s o}$. Note that a break occurs in the Lorentz factor of the shocked jet once the outflow reaches the radius $r=r_{\text {cross }} \approx 3 \times 10^{17} \mathrm{~cm}$ (observer time $t \approx t_{\mathrm{j}}$ ) at which the reverse shock has crossed entirely through the initial shell. The break in $\Gamma_{\mathrm{sh}}$ from this transition occurs at a somewhat larger radius $r \approx 7 \times 10^{17} \mathrm{~cm}\left(t \approx 2 t_{\mathrm{j}}\right)$, once the rarefaction wave launched at $r \approx r_{\text {cross }}$ reaches the forward shock (from MGM12).

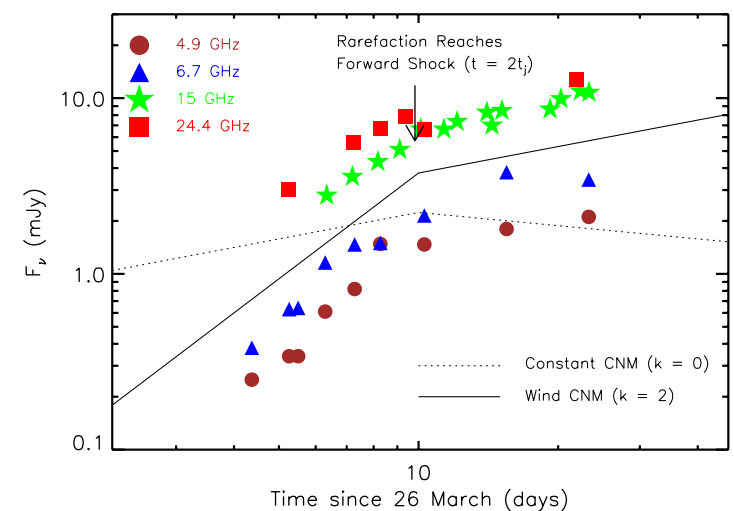

Figure 3. Radio light curves of Swift J1644+57 from Zauderer et al. (2011) at several frequencies below the self-absorption break. The achromatic break observed at $t \sim 10$ days occurs when the rarefaction wave (produced once the reverse shock crosses the initial shell of ejecta) catches up to the forward shock. Shown for comparison are the predicted light curves (arbitrary normalization) if the CNM density has a constant (dotted line; $\mathrm{k}=0$ ) or a wind-type (solid line; $\mathrm{k}=2$ ) radial profile (from MGM12).

MGM12 show that for a CNM of density profile $n_{\mathrm{cnm}} \propto r^{-k}$, the flux below the self-absorption frequency obeys $F_{v<v_{\mathrm{s}}} \propto t^{(k+2) /(4-k)}\left[\propto t^{(k-1) /(4-k)}\right]$ during and much after the RS crossing, respectively. The observed pre- and 
post-break slopes are that both broadly consistent with a wind-type CNM $(k=2)$, as was also found by Zauderer et al. (2011) based on independent arguments. Note that this is just the density profile sampled by the jet on the radial scales $0.01 \mathrm{pc} \lesssim r \lesssim 0.1 \mathrm{pc}$ probed by the first $\sim$ week of observations.

\subsection{Derived properties of the jet and CNM}

If one assumes that by day $23\left(t \approx 6 t_{\mathrm{j}}\right)$ the jet has approached the self-similar BM76 evolution and continues to propagate into a $n_{\mathrm{cnm}} \propto 1 / r^{2}$ medium, then the observed characteristic synchrotron frequency $v_{\mathrm{m}}(t=23 \mathrm{~d}) \equiv v_{\mathrm{m}, 23} \approx 40 \mathrm{GHz}$, self-absorption frequency $v_{\mathrm{sa}}(t=23 \mathrm{~d}) \equiv v_{\mathrm{sa}, 23} \approx 10 \mathrm{GHz}$ and the $4.9 \mathrm{GHz}$ flux $F_{v=4.9 \mathrm{GHz}}(t=23 \mathrm{~d}) \equiv F_{4.9,23} \approx 2 \mathrm{mJy}$ can be solved for the fraction $\epsilon_{e}$ of the shocked energy placed into ultrarelativistic electrons; the number density $n_{18}$ at $r=10^{18}$ $\mathrm{cm}$; and the initial (unshocked) Lorentz factor of the jet $\Gamma_{\mathrm{j}}$ :

$$
\begin{gathered}
\epsilon_{\mathrm{e}}=0.12 \epsilon_{\mathrm{B},-2}^{-1 / 4}\left(\frac{v_{\mathrm{m}, 23}}{10 \mathrm{GHz}}\right)^{1 / 2}\left(\frac{v_{\mathrm{m}, 23}}{10 \mathrm{GHz}}\right)^{1 / 2} \\
n_{18}=1.5 \mathrm{~cm}^{-3} \epsilon_{\mathrm{B},-2}^{-3 / 8}\left(\frac{v_{\mathrm{m}, 23}}{40 \mathrm{GHz}}\right)^{5 / 12}\left(\frac{v_{\mathrm{sa}, 23}}{10 \mathrm{GHz}}\right)^{5 / 6} \\
\Gamma_{\mathrm{j}}=17 \epsilon_{\mathrm{B},-2}^{5 / 32}\left(\frac{v_{\mathrm{m}, 23}}{40 \mathrm{GHz}}\right)^{-1 / 16}\left(\frac{v_{\mathrm{sa}, 23}}{10 \mathrm{GHz}}\right)^{-5 / 8}\left(\frac{F_{4.9,23}}{2 \mathrm{mJy}}\right)^{-1 / 2},
\end{gathered}
$$

where $\epsilon_{B}$ is the fraction of the post-shock thermal energy in the magnetic field and we have assumed a jet with opening angle $\theta_{\mathrm{j}}=1 / \Gamma_{\mathrm{j}}$ (MGM12).

Equation (1)-(3) show that for $\epsilon_{\mathrm{B}} \approx 10^{-3}-0.1$ (as found in many GRB afterglows), one finds reasonable values for $\epsilon_{\mathrm{e}} \approx 0.05-0.2, n_{18} \approx 0.3-10$, and $\Gamma_{\mathrm{j}} \simeq$ $10-20$. The value of $\epsilon_{\mathrm{e}} \approx 0.1$ is similar to those found in GRB afterglows, while $n_{18}$ is somewhat lower than the gas density at a similar location in our own Galactic center near $\operatorname{SgrA}^{\star}$. Since the jet Lorentz factor $\Gamma_{\mathrm{j}}$ is particularly robust, this constrains the beaming fraction of Sw J1644+57 to be $f_{\mathrm{b}} \sim 1 / 2 \Gamma_{\mathrm{j}}^{2} \approx 1-5 \times 10^{-3}$.

\section{DISCUSSION}

\subsection{Implications of jet beaming}

The small beaming fraction $f_{b} \sim 3 \times 10^{-3}$ inferred for Swift $\mathrm{J} 1644+57$ has several implications. First, it implies that the true beaming-corrected peak luminosity of the prompt $\mathrm{X}$-ray $/ \gamma$-ray emission may be as low as $\sim 10^{45} \mathrm{erg} \mathrm{s}^{-1}$, similar to the Eddington luminosity of a $10^{7} M_{\odot} \mathrm{SMBH}$. On the other hand, the beaming-corrected energy of the initial jet was $\sim 10^{51} \mathrm{ergs}$, which requires a jet efficiency of $\epsilon_{\mathrm{j}} \approx 10^{-2}$ (fraction of accretion power placed into the jet) if Swift J1644+57 was indeed powered by the accretion of a solar-mass star. The beaming fraction we infer also appears to be larger than that required to reconcile the rate of J1644-like events with the TDE rate inferred by independent means (e.g. van Velzen et al. 2011) by a factor of $\sim 10-30$ (Burrows et al. 2011). This hints that only a small fraction of TDE events are accompanied by [detectable] relativistic outflows.

The beaming-corrected luminosity of Sw J1644+57 also has implications for the physics of relativistic jet formation. AGN jets are generally thought to be powered by a strong magnetic field which threads the black hole accretion disk or ergosphere. Since the magnetic flux through the initial star is $\sim B_{\star} R_{\star}^{2}$, then flux freezing results in a field near the BH horizon $\left(r \sim 2 R_{g}\right)$ of strength $B_{\mathrm{BH}} \sim B_{\star}\left(R_{\star} / 2 R_{g}\right)^{2}=40 B_{\star, 3} M_{6}^{-2} \mathrm{G}$, where $R_{\star} \sim R_{\odot}$ and we have normalized the stellar field to an (optimistic) value of $1 \mathrm{kG}$. If the jet is dominated by Poynting flux near its base, then to order-of-magnitude the resulting jet power is

$$
L_{\mathrm{j}} \sim \pi\left(2 R_{g}\right)^{2}\left(B_{\mathrm{BH}}^{2} / 4 \pi\right) c \sim 10^{36} B_{\star, 3}^{2} M_{6}^{-2} \mathrm{ergs}^{-1} .
$$

Comparing this expression to the (beaming-corrected) peak X-ray luminosity $L_{X} \sim 10^{45} \mathrm{ergs}^{-1}$ of Swift J1644+ 57 illustrates that the initial field of the disrupted star is by itself $\gtrsim 4$ orders of magnitude too low for any reasonable value of $M_{\mathrm{BH}}$ (Bloom et al. 2011).

A key unsolved theoretical issue in the physics of jet formation is whether the large scale magnetic field required to power the jet is advected from the outer boundary of the flow (e.g. Spruit \& Uzdensky 2005), or whether it is generated locally in the disk by instabilities or dynamo action. Because the magnetic field strength of a solar-type star is insufficient to drive a jet as powerful as Swift J1644+57, this suggests that locally-generated fields may be responsible for the jet. Note that spontaneous large-scale poloidal field generation is not found in current MHD jet simulations, which instead find that a powerful jet of the magnitude required to explain Swift J1644+57 is not produced if the initial poloidal field is weak (e.g. De Veillers et al. 2005; Penna et al. 2010). If such a dynamo is indeed more efficient than found in current simulations and if observations show that only a fraction of (otherwise similar) tidal disruptions are accompanied by a jet would, this would suggest that a second parameter, such as the spin of the black hole, controls the jet strength.

\subsection{Mystery of the radio rebrightening}

Berger et al. (2012) present updated radio light curves of Sw J1644+57, extending up to 6 months after the trigger. Surprisingly, they find that the radio emission rebrightens at all frequencies on a timescale $t \sim 1$ month. This behavior deviates from that expected if the blast wave evolves with constant energy, possibly suggesting that additional energy is contributing to the forward shock at late times (up to a factor 10). B12 proposed that this extra energy results from slower material catching up to the forward shock at late times. Alternatively, the radio brightening could indicate the jet has angular structure, with a high $\Gamma_{\mathrm{j}}$ core dominating the early high energy and radio emission; this could be surrounded by a widerangle, lower $\Gamma_{\mathrm{j}}$ sheath, which dominates the late radio emission. Multidimensional simulations of the jet-CNM interaction, including the possibility of a complex jet structure, are needed to explore these various possibilities 


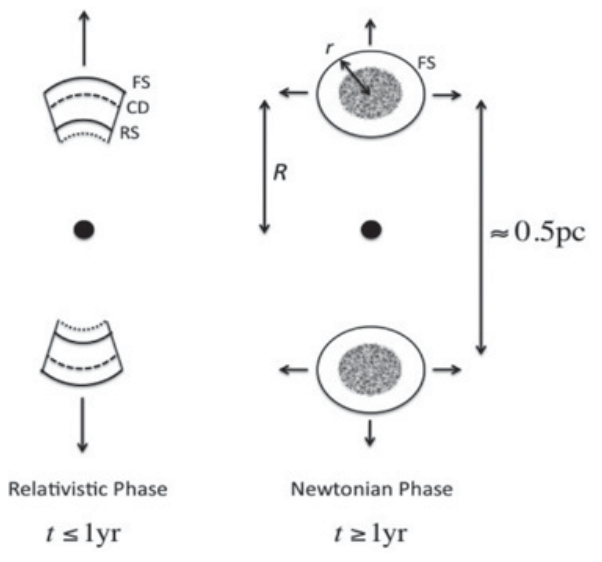

Figure 4. Sketch of the ultra-relativistic (left) and Newtonian (right) stages of jet deceleration. Initially, the jet propagates radially, forming a forward/reverse shock structure (Fig. 1), during which time the radio emission is relativistically beamed along the direction of the jet axis. Once the configuration has slowed to non-relativistic speeds, each of the two jet lobs expands quasi-spherically. For a typical off-axis viewer, emission peaks at the transition between the relativistic and non-relativistic stages (from GM11).

in detail. This is a particularly important exercise because the opening angle and energy in moderate- $\Gamma_{\mathrm{j}}$ material affects the likelihood of detecting events similar to $\mathrm{Sw}$ $\mathrm{J} 1644+57$ viewed off-axis (see below).

\subsection{Blind detection with future radio surveys}

After the blast wave decelerates to mildly relativistic speeds, the jet begins to spread laterally (Zhang \& MacFadyen 2009), eventually relaxing into a nonrelativistic, spherical expansion (Sedov-Taylor phase) centered at the location of the deceleration of the blast (Fig. 4). Radio emission becomes isotropic following this non-relativistic transition, in which case it could be visible even in cases where the initial high energy emission was missed because the jet was pointing in a different direction.

Radio astronomy is poised for a revolution in the study of time-domain ('transient') phenomena over the coming decade, with new wide-field survey arrays coming online at both meter wavelengths (e.g. LOFAR, MWA, and LWA) as well as GHz frequencies (e.g. Apertif/WSRT, MeerKAT, and ASKAP). Despite the overall maturity of the field of radio astronomy, surprisingly little is known about the radio transient sky. Frail et al. (2012) show that Swift J1644+57-like events viewed off-axis could in fact dominate the $\mathrm{GHz}$ transient sky, with 10100 events potentially detectable per year by upcoming surveys (see also GM11). Future monitoring of Swift $\mathrm{J} 1644+57$ through the non-relativistic phase over the coming years will thus provide us with a better understanding of how similar events would appear to a viewer with a more typical observing angle. If the emission remains bright at high frequencies, the radio lobes may become resolvable with VLBI on a similar timescale. With similar motivation, targeted searches have begun for late radio emission from TDE candidates detected previously at X-ray, UV, or optical wavelengths. Detections or upper limits from these studies will help constrain the diversity of relativistic outflows accompanying TDEs.

\subsection{Reverse Shock Dominated Jets?}

Figure 3 shows how the dynamics of the reverse shock is directly imprinted on the early radio light curve of Swift J1644+57. Despite this dynamical imprint, the radio emission itself is likely dominated by electrons accelerated at the forward shock (as in normal GRB afterglows). Energy radiated by the reverse shock may be less important in the case of Swift J1644+57 than originally anticipated by GM11 because the duration of peak jet activity was relatively short and the CNM density was relatively low. However, this need not universally be the case. For a TDE surrounded by a denser CNM, or producing a jet of longer duration (as might arise for a disrupted star with a longer fall-back timescale), the RS extracts a larger fraction of the jet power and itself may come to dominate the early light curve (an extreme version of the 'thick shell' limit of normal GRB afterglows). Since the jet also decelerates rather abruptly in this case (possibly directly to nonrelativistic speeds and jet spreading, even on a relatively short timescale $\lesssim t_{\mathrm{j}}$ ), this may lead to radical changes in both on- and off-axis light curves. Numerical studies of this novel regime of 'reverse shock dominated' jet-CNM interaction thus represent an intriguing avenue for future research.

\section{References}

[1] Ayal, S., Livio, M., \& Piran, T. 2000, ApJ, 545, 772

[2] Berger, E., Levan, A., Tanvir, N. R., et al. 2011, GRB Coordinates Network, 11854, 1

[3] Berger, E., Zauderer, A., Pooley, G. G., et al. 2012, ApJ, 748, 36

[4] Blandford, R. D., \& McKee, C. F. 1976, Physics of Fluids, 19, 1130

[5] Bloom, J. S., Giannios, D., Metzger, B. D., et al. 2011, Science, 333, 203

[6] Burrows, D. N., Kennea, J. A., Ghisellini, G., et al. 2011, Nature, 476, 421

[7] De Villiers, J.-P., Hawley, J. F., Krolik, J. H., \& Hirose, S. 2005, ApJ, 620, 878

[8] Giannios, D., \& Metzger, B. D. 2011, MNRAS, 416, 2102

[9] Guillochon, J., Ramirez-Ruiz, E., Rosswog, S., \& Kasen, D. 2009, ApJ, 705, 844

[10] Levan, A. J., Tanvir, N. R., Cenko, S. B., et al. 2011, Science, 333, 199

[11] Lodato, G., King, A. R., \& Pringle, J. E. 2009, MNRAS, 392, 332

[12] Lodato, G., \& Rossi, E. M. 2011, MNRAS, 410, 359

[13] Penna, R. F., McKinney, J. C., Narayan, R., et al. 2010, MNRAS, 408, 752

[14] Rees, M. J. 1988, Nature, 333, 523

[15] Spruit, H. C., \& Uzdensky, D. A. 2005, ApJ, 629, 960 
[16] Strubbe, L. E., \& Quataert, E. 2009, MNRAS, 400, 2070

[17] Ulmer, A. 1999, ApJ, 514, 180

[18] van Velzen, S., Farrar, G.R., Gezari, S., et al. 2011, ApJ, 741, 73
[19] van Velzen, S., Körding, E., \& Falcke, H. 2011, MNRAS, 417, L51

[20] Zauderer, B. A., Berger, E., Soderberg, A. M., et al. 2011, Nature, 476, 425

[21] Zhang, W., \& MacFadyen, A. 2009, ApJ, 698, 1261 